E.A. Yushkov

\title{
MODELING OF OPERATION MODES OF SHIP POWER PLANT OF COMBINED PROPULSION COMPLEX WITH CONTROL SYSTEM BASED ON ELECTRONIC CONTROLLERS
}

Purpose. Designing of diagrams to optimize mathematic model of the ship power plant (SPP) combined propulsion complexes (CPC) for decreasing operational loss and increasing fuel efficiency with simultaneous load limiting on medium revolutions diesel generator (MRDG) by criterion reducing of wear and increasing operation time between repairs. Methodology. After analyzing of ship power plant modes of CPC proposed diagrams to optimize mathematic model of the above mentioned complex. The model based on using of electronic controllers in automatic regulation and control systems for diesel and thruster which allow to actualize more complicated control algorithm with viewpoint of increasing working efficiency of ship power plant at normal and emergency modes. Results. Determined suitability of comparative computer modeling in MatLab Simulink for building of imitation model objects based on it block diagrams and mathematic descriptions. Actualized diagrams to optimize mathematic model of the ship's power plant (SPP) combined propulsion complexes (CPC) with Azipod system in MatLab Simulink software package Ships_CPC for decreasing operational loss and increasing fuel efficiency with simultaneous load limiting on medium revolutions diesel generator (MRDG) by criterion reducing of wear and increasing operation time between repairs. The function blocks of proposed complex are the main structural units which allow to investigate it normal and emergency modes. Originality. This model represents a set of functional blocks of the components SPP CPC, built on the principle of «input-output». For example, the function boxes outputs of PID-regulators of MRDG depends from set excitation voltage and rotating frequency that in turn depends from power-station load and respond that is a ship moving or dynamically positioning, and come on input (inputs) of thruster rotating frequency PID-regulator models. Practical value. The results of researches planned to use in creation of software package Ships_CPC, in Mat Lab/Simulink was developed under the state budget project "Concepts, technologies and ways of improving ship plants combined propulsion complexes» at the Department of Electromechanics and Electrical Engineering of National University "Odessa Maritime Academy» (State registration number 0114u000340). References 9, figures 6.

Key words: ship power plants, combined propulsion complex, mathematical modeling, electronic controller, thruster, diesel, dynamic positioning.

В результате анализа режимов работы судовой энергетической установки комбинированного пропульсивного комплекса предложены схемы оптимизации математической модели указанного комплекса. Модель основана на применении электронных регуляторов в системах автоматического регулирования и управления дизелем и подруливающим устройством, которые позволяют реализовать более сложные алгоритмы управления с точки зрения повышения эффективности работы судовой энергетической установки при нормальных и аварийных режимах. Библ. 9 , рис. 6.

Ключевые слова: судовая энергетическая установка, комбинированный пропульсивный комплекс, математическое моделирование, электронный регулятор, подруливающее устройство, дизель, динамическое позиционирование.

Introduction. In recent years rowing power plants (RPP) are the best solutions for certain types of ships. The so-called combined propulsive systems (CPS) with electric propulsion motors on shaft line are variable speed throughout the range of coordinates with direct control moment and powered on based on many tire designs flexible systems AC uneven distribution impedance $[1,2]$ which are the only ones with multiple power grids diesel-generators or turbo-generators which have found many advantages in several areas of shipbuilding. Fuel savings, reduced maintenance costs, improved flexibility, high reliability, reduced noise and vibration this is the main thing that marks these systems on the market today. An additional start-up costs associated with the increase in the number of components does not negate these advantages, because such systems are very flexible in terms of operation, management and placement on board. Electrical equipment also shows high efficacy in a large range of operational modes. But to take advantage of such systems today require study of existing models of RPP SPC, developing new models and management strategies, analysis and optimization of marine vessels operating in various operating conditions [3], particularly on hold position $(D P)$.
Problem definition. Detailed mathematical models of RPP SPC are presented and studied very carefully cont recent years [4], where the emphasis is given to mediumtorque diesel generators (MTDG). We know that ship power systems that have stringent requirements for voltage and frequency so that MTDG models developed from this review have no prospects for change. They differ in power and consumption of power.

Frequency converters (FC), thruster ( $\mathrm{T}$ ) and other internal load include functional blocks of the power consumption of the overall distribution of active and reactive power. PID-controllers of MTDG, T and automatic voltage regulators (AVR) checked in terms of performance [5], and it turned out that it is enough for simulations that cover the main indicators of supply ship power plant (SPP) CPS but not enough to account degradation effects occurring on lines shaft $[6,7]$.

At present the technical operation of such systems are the following problems:

- compliance with the requirements of quality management (Failure modes and effects analysis - FMEA) encountered during operation; 
- unification of power distribution management system (Power Management System - PMS) in combination functions in relation to other similar;

- independence components of PMS from each other, even to the level sensors;

- not only reduce power in the calculation of the total estimated load, but also load single generator;

- conformity of the conditions of increased pressure in terms of adequacy to ensure the normal operation depending on any abnormal situation and reloading ship power system (SPS) in general.

To solve the above problems it is necessary, based on theoretical assumptions regarding electronic controllers and models of heat engines and maneuvering propulsion device, design a circuit optimization mathematical SPP SPC which would allow to implement more complex control algorithms in terms of improving the efficiency of SPP for the entire range load.

The goal of the paper is the development of schemes of optimization mathematical model of SPP SPC with screw-type Azipod steering installation to reduce operating costs and improve fuel efficiency while loading restriction on the criterion MTDG reduce wear and increase turnaround time.

MTDG are generally designed for short-term overload up to $115 \%$ of nominal. Power generation units located on three in two engine rooms (ER). Each ER has an independent system of support mechanisms that include fuel system, air pressure and low cooling water that are able to cross-connect. Two groups of three MTDG are connected to the two main sections of tires of high voltage. Section interconnected sectional switch.

From the main switchboard (MSB) by reducing the high voltage transformers will supply switchboards (SSB) of own needs and transformers for power supply to electric technology inherent in this type of vessel. Low voltage distribution system consists of two main tires left and right sides at the main deck, which are fed from the main transformer high-voltage interconnected switches that interlocked with the transformer feeder breakers.

Each MTDG is equipped by independent system of control and regulation of power (Fig. 1). Complete switching SODH include: relays generator, digital module synchronization and load distribution, $P L C$ with interface $\mathrm{I} / \mathrm{O}$, power converters and remote control panel with alarm. MTDG are staffed by management system (Electro-Motive Diesel Engine Control system - EMDEC) which provides speed control with closed loop, injection, start/stop function and alarm. EMDEC MTDG is operating from the 24/48 V DC dedicated battery/charger.

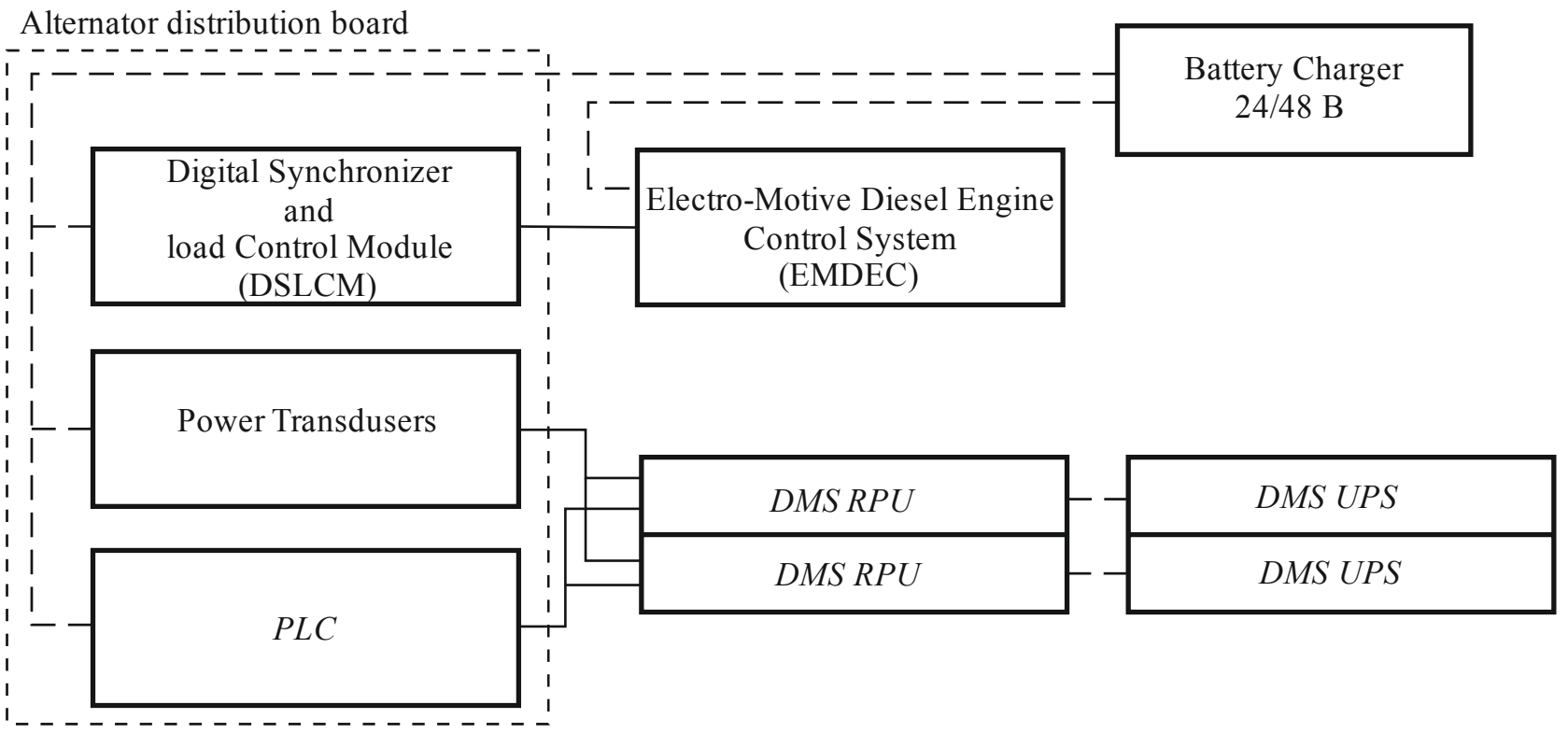

Fig. 1. MTDG control system configuration: $P L C$ - programmed logic controller; $D M S$ - data management system; $R P U-$ reserve power unit; UPS - uninterruptible power supply

MTDG can be run from the front panel in manual mode or remote mode «Auto» by the system DMS. Distribution $P L C$ controls the functions of the engine, and the digital module synchronization and load control DSLC controls the timing and distribution of power.

$D P$ system is double-redundant (Fig. 2). Two main operator consoles $(A S K 1, A S K 2)$ contain control processors, keyboards, control and display. DP system can control up to eight engines $T$, as usual - to four. Control console located in the chassis scars, which also has three signal processing devices (SPU1, SPU2, SPU3), which are the devices $\mathrm{I} / \mathrm{O}$ signals from the power position sen- sors, gyroscopes, sensors and wind movement. Each individual unit has an independent channel of communication with the main central control computer.

Two signal processing units (SPU4 and SPU5) located at the MSB carry out signal processing function power management on two backup devices interface I/O.

As sources of electricity in handheld power plant salient-pole brushless synchronous generators (SG) of high voltage with diesel- or turbo-drive are used. The most convenient form of mathematical models of these machines is machines in the form of an orthogonal coordinate system $d, q$ which is rigidly connected to the rotor 
and rotates with it with a synchronous speed [8]. This coordinate system has advantages over a fixed coordinate system $a, b, c$ in which, during rotation of the rotor axis of the rotor and stator windings mutually moved because phase AC voltage, current and flux expressed in periodic variables. Vectors voltage, current and flux in the perpendicular arranged axes $d, q$ are constant and relatively fixed axles, which excludes mutual between them and simplifies the analysis model. The disadvantages of modeling in axes $d, q$ include the inability to study asymmetric modes. In order to improve clarity and simplify the simulation results analysis more convenient to use equations in relative units.

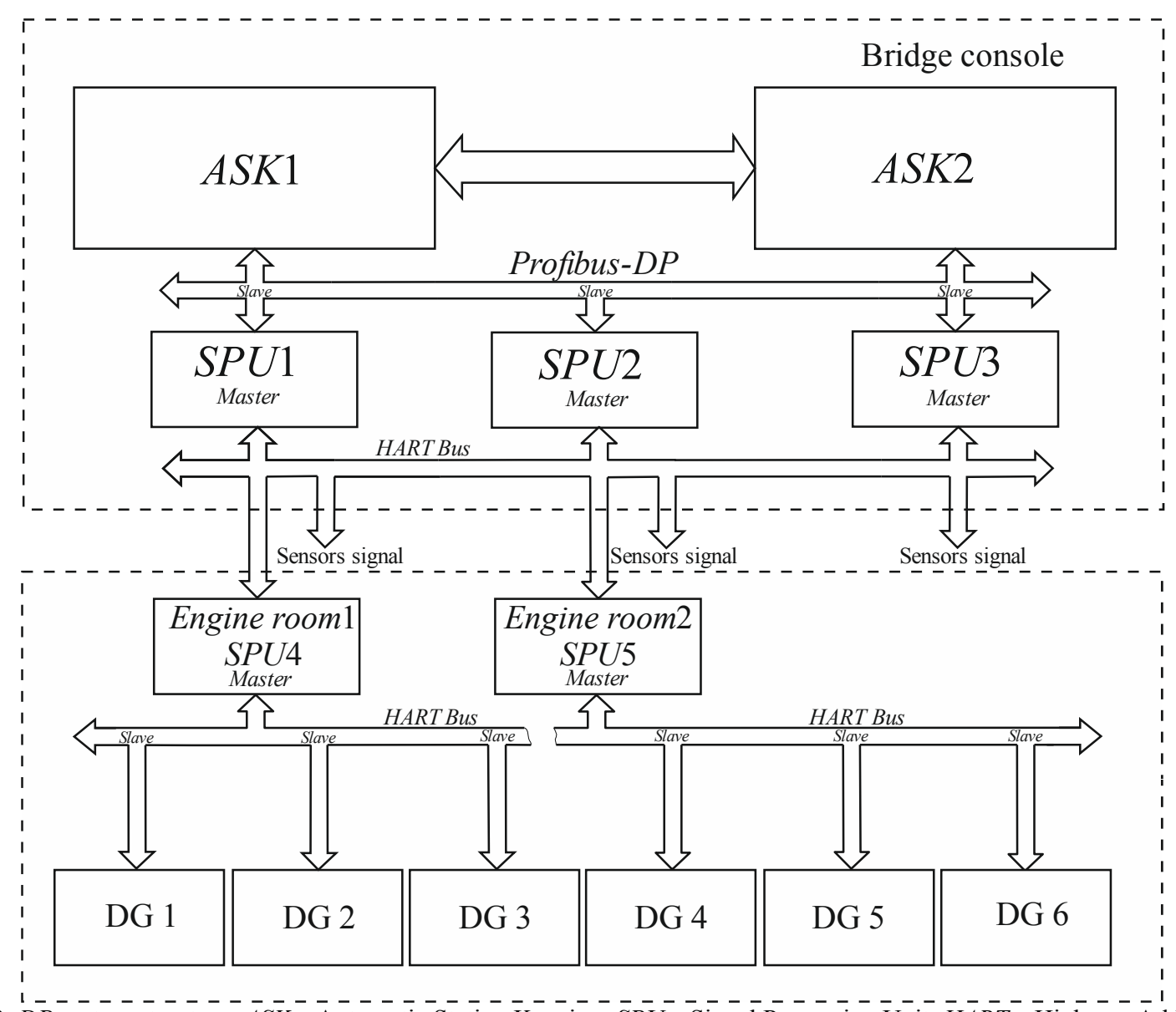

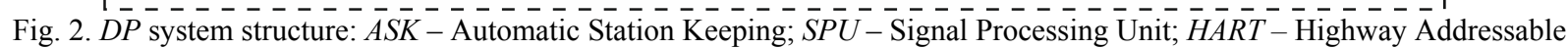
Remote Transducer Protocol

For rigid framework of fixed voltage and frequency for the MSB and SSB used for standard voltage and power factor on modular tires. Ship power system is isolated and models presented in this article, a rotor speed SG chosen as the base, and viewing other power generators are calculated in relation to it. The angle of the base power generator and the voltage on the tire load module is calculated according to the current generator.

For each of the SG model of the 7th order is used, and the relationship between generators through load module that represents the overall burden as AC impedance, which makes the vector components of the load to the output current generators and voltage vector returns on tires depending on the load characteristics (constant impedance, constant power, etc.). Electric values are modeled in axes $d, q$. For example, one model MTDG model consists of diesel and SG, PID speed control diesel and $A V R$. Function on/off for each part MTDG model simulations load connecting/disconnecting this unit to the MSB. The scheme of the general structure of the model with input and output parameters is shown in Fig. 3.

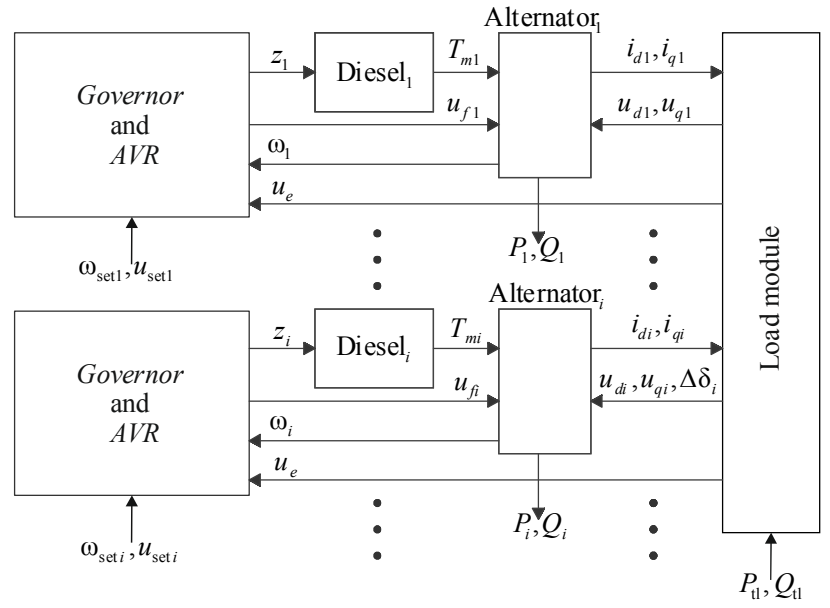

Fig. 3. Flowchart of the ship power plant model: $z$ - fuel index, relative units.; $T_{m}$ - diesel time constant, $\mathrm{s} ; u_{f}$ - excitation voltage setpoint, relative units; $\omega-$ MTDG shaft rotation speed, $\mathrm{rad}^{-}$ ${ }^{1} ; i_{d}, i_{q}, u_{d}, u_{q}$ - current and voltage components by corresponding axes, relative units; $P, Q$ - active and reactive powers, relative units.; $\Delta \delta$ - load angle, rad 
Developing an AVR mathematical model based on electronic PID-controller and the power semiconductor switch, consider their characteristics [9]. Appointment proportional integral differential (PID) controller is to maintain the specified value $u_{\text {set }}$ some input values through its comparison with the initial current value which is made by negative feedback signal $u_{\mathrm{fb}}$. The difference between these two values is called inconsistency or deviation from the set value. The power semiconductor switch (PSS) of AVR serves as a key between a source of constant tension and winding brushless exciter. Voltage control pathogen carried by regulating the duration included (disconnected) state of the key semiconductor, i.e. by pulse width submitted to the excitation winding of the exciter. This type of regulation is called pulse width (PWM), and the PSS have a switching frequency of 10 $\mathrm{kHz}$. In general, the power converter is nonlinear dynamic link. Since the switching frequency is much higher than the bandwidth of the system, the power converter can also be viewed as lagless link. As a link in the system of regulation, the PSS is usually described aperiodic link or link with a delay.

For normal AVR operation must be specified limit values of the output voltage to prevent situations overexcited and under exited generator. Block restrictions - a stabilizing element. Also, the model must be supplemented AVR element that describes a measuring alternating voltage generator in DC signals adapted for PID controller. The converter converts the voltage signals into digital instrument transformers, its transfer function can also be expressed in terms of aperiodic link of the first order.

Regulatory parameter MTDG drive motor as the object of the automatic control system (ACS) is the speed of its shaft. The moment of the engine, offset by losses and moment resistance point generator, which prevent rotation of the shaft. Torque engine depends on the amount of fuel which enters it, thus regulating effect on the frequency of rotation of the motor shaft creates a regulatory body that changes the amount of fuel. For diesel this regulatory body is rail of the high-pressure fuel pumps (HPFP). In ship MTDG producing alternating current to drive sustainability rotational speed of the diesel engine are presented the most stringent requirements, provided that the requirements of regulations to ship frequency alternating current network, so you need to support high-speed diesel drive mode with high accuracy, irrespective of changing the ship's electrical load power. For this purpose, the system of automatic speed control. Currently, the ships of the old buildings are used in mechanical and hydromechanical regulators rotational speed diesel engines that have proven to be reliable working regulating device. However, the functionality of these controllers are limited, therefore improving modern automatic control systems (ACS) and systems of automatic control (SAC) ship diesel engines is moving towards the use of electronic controls based on microprocessor.

With the use of electronic controls in ACS and SAC diesel engine provided a new, higher level, allowing you to implement more complex control algorithms diesel and provide previously unattainable performance quality of the regulation of its speed. A prerequisite for this process of improving the quality of regulation is to optimize how the structure of the regulator and its parameters. Choosing the structure of regulatory law and regulation is determined by several factors. In modern SPP SPC MTDG equipped with digital PID-control speed. In addition, you must also add the block, describing servomechanism HPFP rails, as which can be used executive electric and electrohydraulic units. In this case, the most convenient is to describe this item using aperiodic links of the first order. The very same diesel engine introduces some delay after repositioning rail fuel pump to a corresponding change in the frequency of rotation of its shaft is connected with the time on the course of chemical processes inside the combustion chamber, as well as the inertia of mechanical assemblies. Because it is most preferably diesel seems like pure link delay.

Results of investigations. Structural models of electronic AVR and regulator speed diesel according to Fig. 3 , based on mathematical models of its key elements, according to the above conditions, are presented in Fig. 4.

The modeling will be done in MatLab Simulink, and, given the results of studies, dependence and transfer functions control speed $\mathrm{T}$ [5] operating in different modes that correspond to a particular operating mode of the ship in general, try to get the graphics speed shaft $\mathrm{T}$ and power consumption as a function of time and confirm the adequacy of mathematical models. To do this, use a software package Ships_CPC developed as part of the research state budget of «Concepts, technologies and areas of improvement ship power plants combined propulsive complexes» of the National University (Odessa Maritime Academy), which is a set of functional blocks of the components of SPP SPC built on a classic the principle of «input-output». For example, according to the block diagram in Fig. 3, the outputs of functional blocks PIDregulators MTDG dependent on given excitation voltage and speed, which in turn depends on the load on the power that corresponds to a moving boat or dynamically positioned, and the input (inputs) models PID-regulators the rotation frequency of $\mathrm{T}$.

SPP SPC consists of $11 \mathrm{kV}$ MSB, switchboards, 5 generators, 3 azimuthal $\mathrm{T}$ with variable speed and several DOL (Direct On Line) motors. The total installed capacity is $40 \mathrm{MW}$, while $\mathrm{T}$ capacity of $3 \times 2.8 \mathrm{MW}$ is powered by frequency converters (FC) of 12-pulse topology. The maximum speed for azimuth thrusters 1000 RPM but in the scheme of the line of shafts are present lowering transmission between the engines and propellers of fixed pitch (PFP) but measurements taken directly from the line of shafts via inductive sensors that provide signals of rotational speed to $D P$-controller.

Simulations using the strategy of vector control and direct control point and power, as described in [5], and flowcharts shown in Fig. 3 and 4 are shown in Fig. 5, and corresponding consumption of T capacity - in Fig. 6.

The simulation results are compared with field measurements for type vessels Supply vessel which performs dynamic positioning. To measure the ship were only available mechanical power $\mathrm{T}$, so the graphs depict modulated depending on electricity consumption and power loss in induction motors T. Temporary delays in the registration of measurement data can be explained by, 
for example, in the same sequence of horizontal there are some differences between simulation and measurement. In other cases, of course, affect the results of tuning con- trollers, especially the controller of rotational speed MTDG and T.

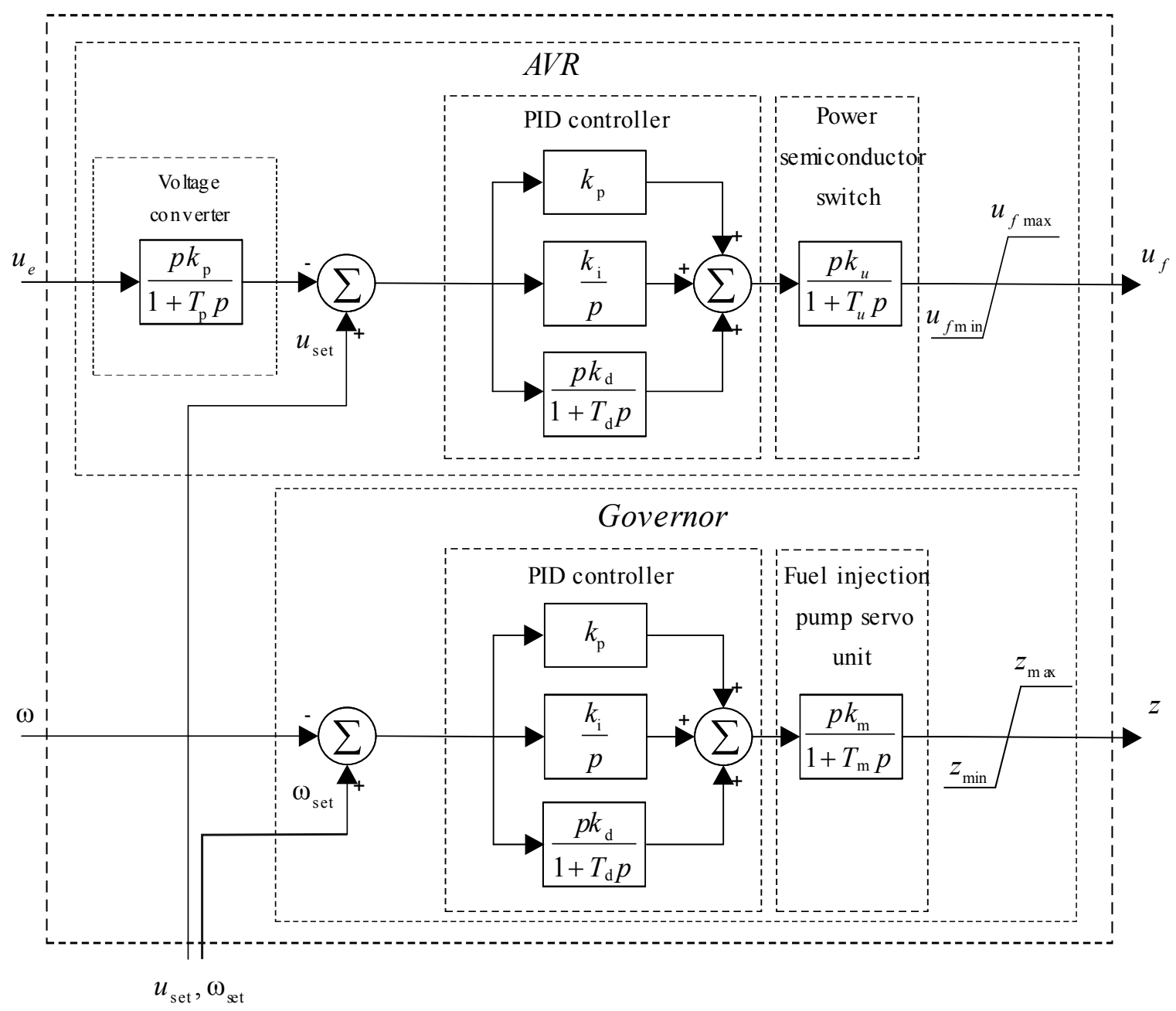

Fig. 4. Flowchart of the MTDF regulators: $k_{\mathrm{p}}, k_{\mathrm{i}}, k_{\mathrm{d}}, k_{\mathrm{m}}, k_{u}$-coefficients of proportional, integrating and differential units respectively, the converter voltage servomotor HPFP and PSS; $T_{\mathrm{p}}, T_{\mathrm{i}}, T_{\mathrm{d}}, T_{\mathrm{p}}, T_{\mathrm{m}}, T_{u}$ - time constants proportional, integrating and differential units respectively, the converter voltage servomotor HPFP and PSS

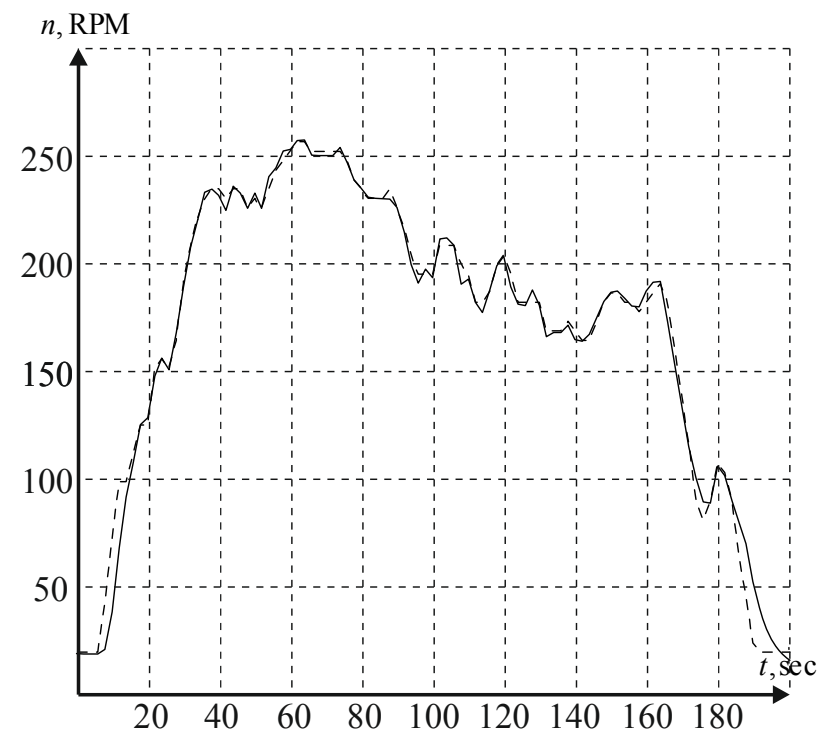

Fig. 5. Measured and modeled T rotation frequency dependencies: --- modelling, - measurement

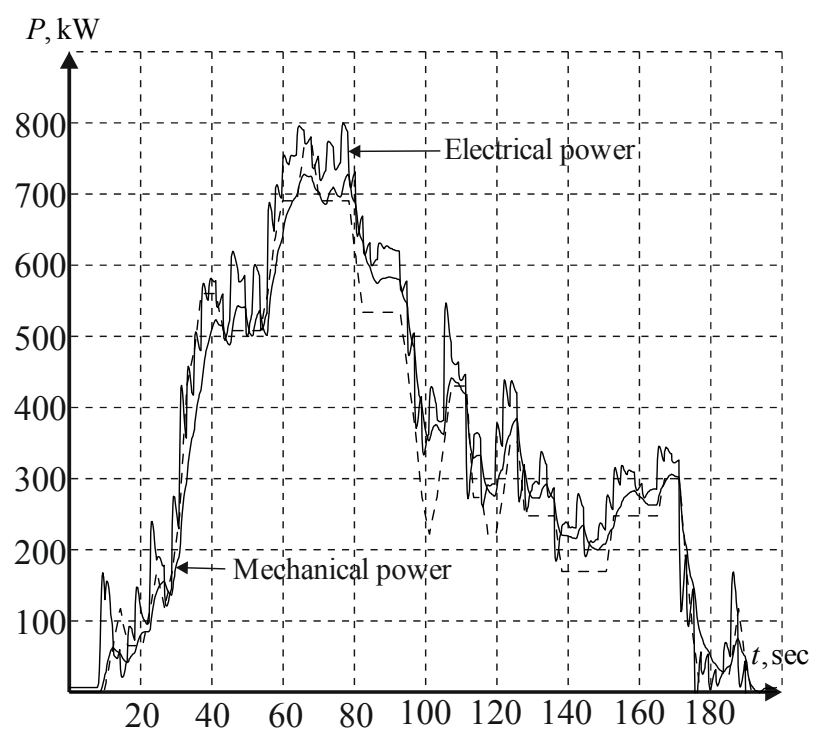

Fig. 6. Measured and modeled T consumption power dependencies: --- modeling, — simulation 
Internal control loops $\mathrm{T}$ (torque, flux and current) are set out as the biggest gain in order to explore the process performance in regulating torque while maintaining the value stream as close to the nominal value for different load conditions.

\section{Conclusions.}

1. Appropriateness of comparative computer simulation in MatLab Simulink to build simulation models of objects based on their block diagrams and mathematical descriptions is defined.

2. In an environment MatLab Simulink optimization schemes mathematical models of MTDG and RPP SPC with screw- steering type Azipod are implemented installing the software complex Ships_CPC, to reduce operating costs and improve fuel efficiency while loading restriction on the criterion of MTDG reduce wear and increase turnaround time.

3. The functional blocks of the proposed complex are basic structural units of RPP SPC models allowing them to investigate the normal and emergency operation modes.

Further studies will be conducted to establish the parameters of mathematical models AVR and frequency of MTDG in the environment MatLab Simulink. The method can be used to preselect the parameters of modern electronic controls. There will also be performed computer simulations of processes in the RPP SPC with different types of $\mathrm{T}$ at: the change of frequency of $\mathrm{T}$ rotation; the reverse; dynamic changes of emphasis propellers; failure of one $\mathrm{T}$; changes of rowing propellers torque. The obtained results should reveal characteristic changes of basic parameters of RPP SPC at the relevant operation modes that will permit to consider investigated models adequate to real objects.

\section{REFERENCES}

1. Woud H.K., Stapersma D. Design of propulsion and electric power generation systems. IMarEST Publications, London, 2003. $494 \mathrm{p}$.

2. Budashko V.V., Onishchenko O.A. Improving management system combined thruster propulsion systems. Bulletin of NTU «KhPI», 2014, no.38(1081), pp. 45-51. (Ukr).
3. Budashko V.V., Onishchenko O.A. Mathematical principles of simulation of power plant's control system at drillship. Bulletin of Kamchatka State Technical University, 2014, no.29, pp. 613. (Rus).

4. Hansen J.F. Modelling and control of marine power systems. Doktor ingeniør thesis, Trondheim, Norway, 2000. 119 p. Available at: http://www.itk.ntnu.no/databaser/dr_ing_avhandlinger/vedlegg/ 110 pdf.pdf (accessed 21 September 2015).

5. Budashko V.V., Yushkov Y.A. Mathematic modeling of allrange controllers speed of thrusters for ship power plants in combined propulsion complexes. Electronic Modeling, 2015, vol.37, no.2, pp. 101-114. (Rus).

6. Glazeva O.V., Budashko V.V. Aspects of the mathematical modelling of the elements for Western Systems Coordinating Council of combined propulsion complexes. Bulletin of NTU "KhPI». Series: Problems of Electrical Machines and Apparatus Perfection. The Theory and Practice, 2015, no.42(1151), pp. 71-75. (Ukr).

7. Bojko A.A., Budashko V.V., Yushkov E.A., Bojko N.A. Synthesis and research of automatic balancing system of voltage converter fed induction motor currents. Eastern-European Journal of Enterprise Technologies, 2016, vol.1, no.2(79), pp. 22-34. doi: 10.15587/1729-4061.2016.60544.

8. Chernikh I.V. SimPowerSystems: Modelirovanie elektrotekhnicheskikh ustroistv i sistem v Simulink (SimPowerSystems: Modeling of electrotechnical devices and systems in Simulink). Available at: http://matlab.exponenta.ru/simpower/book1/2.php (accessed 01 October 2015). (Rus).

9. Tokarev L.N. [Automated regulation systems. Tutorial]. Saint Petersburg, Notabene Publ., 2001. 191 p. (Rus).

Received 09.05.2016
E.A. Yushkov, Postgraduate Student,
Odessa National Maritime Academy,
8, Didrikhson Str., Odessa, 65029,
phone +38 048 7332367, e-mail: ushkov@te.net.ua

How to cite this article:

Yushkov E.A. Modeling of operation modes of ship power plant of combined propulsion complex with control system based on electronic controllers. Electrical engineering \& electromechanics, 2016, no.6, pp. 39-44. doi: 10.20998/2074272X.2016.6.07. 